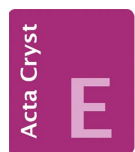

CRYSTALLOGRAPHIC COMMUNICATIONS

ISSN 2056-9890

Received 29 July 2017

Accepted 3 August 2017

Edited by J. Simpson, University of Otago, New Zealand

Keywords: crystal structure; sulfonamide herbicides; triazolopyrimidine herbicides; penoxsulam.

CCDC reference: 1566461

Supporting information: this article has supporting information at journals.iucr.org/e

\section{Crystal structure of penoxsulam}

\author{
Hyunjin Park, Jineun Kim,* Hojae Chiang and Tae Ho Kim*
}

Department of Chemistry (BK21 plus) and Research Institute of Natural Sciences, Gyeongsang National University, Jinju 52828, Republic of Korea. *Correspondence e-mail: thkim@gnu.ac.kr, jekim@gnu.ac.kr

The title compound, $\mathrm{C}_{16} \mathrm{H}_{14} \mathrm{~F}_{5} \mathrm{~N}_{5} \mathrm{O}_{5} \mathrm{~S}$ [systematic name: 2-(2,2-difluoroethoxy)$N$-(5,8-dimethoxy-1,2,4-triazolo[1,5-c]pyrimidin-2-yl)-6-(trifluoromethyl)benzenesulfonamide], is used as a herbicide. The asymmetric unit of this structure comprises two independent molecules, $A$ and $B$. The dihedral angles between the ring planes of the triazolopyrimidine ring systems and the benzene rings are $68.84(7)^{\circ}$ for $A$ and $68.05(6)^{\circ}$ for $B$. In the crystal, weak intermolecular $\pi-\pi$ interactions, with centroid-centroid separations of $3.4456(17)$ and $3.5289(15) \AA$ and $\mathrm{C}-\mathrm{F} \cdots \pi\left[3.5335(17) \AA\right.$ and $\left.107.92(13)^{\circ}\right]$ contacts link adjacent molecules into chains along [001]. $\mathrm{C}-\mathrm{H} \cdots \mathrm{O}$ and $\mathrm{C}-$ $\mathrm{H} \cdots \mathrm{F}$ hydrogen bonds link type $B$ molecules into chains parallel to (100). Additional $\mathrm{C}-\mathrm{H} \cdots \mathrm{F}$ hydrogen bonds together with short $\mathrm{F} \cdots \mathrm{F}$ contacts further aggregate the structure into a three-dimensional network.

\section{Chemical context}

Penxosulam is a triazolopyrimidine sulfonamide herbicide, which is used to control the growth of annual grasses, sedges, and broadleaf weeds in rice agriculture. The compound inhibits the synthesis of acetolactate and targets the biosynthesis of branch-chained amino acids, a metabolic pathway found in plants, fungi, and microorganisms. Acetolactate synthase (ALS) inhibitors are present in most effective herbicides. They are used in agriculture because they show a broad weed control spectrum, crop selectivity, are safe to humans, and can be applied at relatively low usage rates (Jabusch et al., 2005; Yasuor et al., 2009). Moreover, penoxsulam controls a number of troublesome weeds including northern jointvetch, alligatorweed, Texasweed/Mexicanweed, annual sedge, ducksalad, smartweed, and hemp sesbania (Willingham et al., 2008). We now report here the crystal structure of penoxsulam, 2-(2,2difluoroethoxy)- $N$-(5,8-dimethoxy[1,2,4]triazolo[1,5-c]-pyrimidin-2-yl)-6-(trifluoromethyl)benzenesulfonamide.

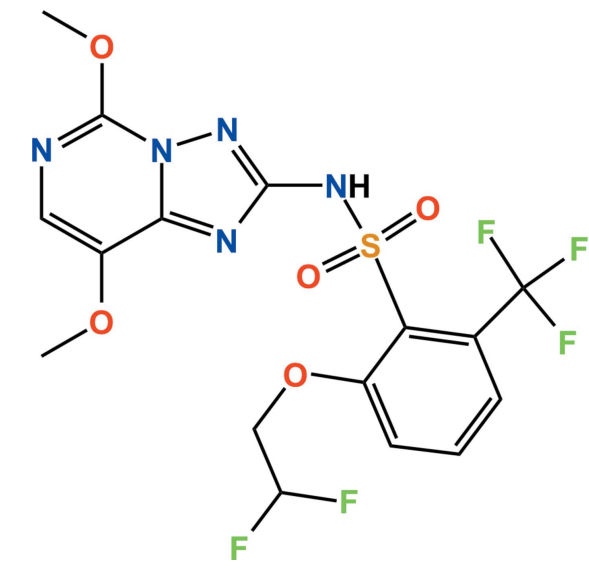




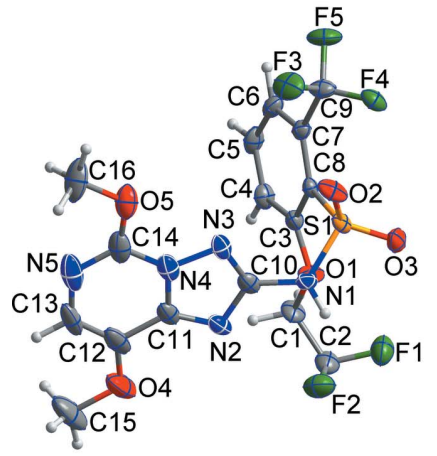

Molecule A

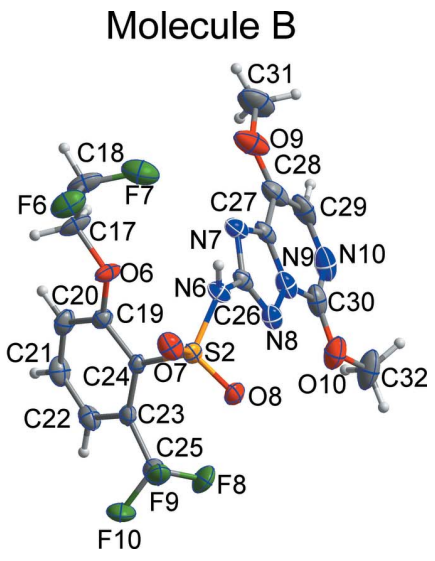

Figure 1

The molecular structures of the title compound with the atom labelling and displacement ellipsoids are drawn at the $50 \%$ probability level. $\mathrm{H}$ atoms are shown as small spheres of arbitrary radius.

\section{Structural commentary}

The penoxsulam molecule crystallizes with two independent molecules, $A$ and $B$ in the asymmetric unit, Fig. 1. The triazolopyrimidin unit caries methoxy substituents while the benzene ring of the benzenesulfonamide segment of the molecule carries trifluoro methyl and the unusual difluoroethoxy substituents. The dihedral angles between the planes of the triazolopyrimidine ring systems and the benzene ring planes are $68.84(7)^{\circ}$ in molecule $A$ and $68.05(6)^{\circ}$ in $B$. All bond lengths and bond angles are normal and comparable to those observed in similar crystal structures for triazolopyrimidine (AboulWafa et al., 2014) and triazolopyrimidine sulfonamide herbicides (Kumar et al., 2012).

\section{Supramolecular features}

In the crystal, there are weak $\pi-\pi$ interactions between the pyrimidine rings of neighbouring molecules of type $A$ with $C g 1 \cdots C g 1^{\mathrm{v}}=3.4456(17)$, and type $B$ with $C g 2 \cdots C g 2^{\mathrm{vi}}=$ 3.5289 (15) $\AA$ [ $C g 1$ and $C g 2$ are the centroids of the N4/N5/ C11-C14 and N9/N10/C28-C30 rings, respectively; symmetry codes: (v) $-x,-y,-z+1$; (vi) $-x+2,-y,-z]$. These combine

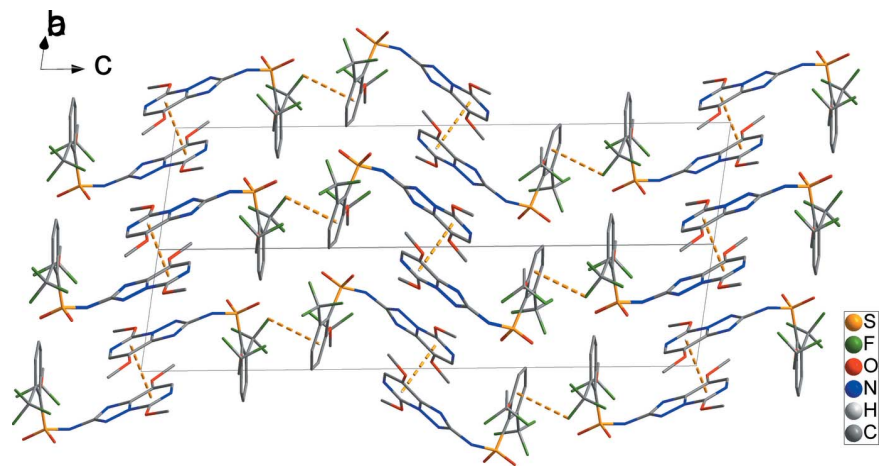

Figure 2

$\mathrm{C}-\mathrm{F} \cdots \pi$ and weak $\pi-\pi$ interactions, yellow dashed lines, form chain along [001] in the crystal packing. $\mathrm{H}$ atoms have been omitted for clarity.
Table 1

Hydrogen-bond geometry $\left(\AA,^{\circ}\right)$.

\begin{tabular}{lllll}
\hline$D-\mathrm{H} \cdots A$ & $D-\mathrm{H}$ & $\mathrm{H} \cdots A$ & $D \cdots A$ & $D-\mathrm{H} \cdots A$ \\
\hline $\mathrm{C} 1-\mathrm{H} 1 A \cdots \mathrm{F} 10^{\mathrm{i}}$ & 0.99 & 2.48 & $3.293(3)$ & 139 \\
$\mathrm{C} 16-\mathrm{H} 16 C \cdots \mathrm{F} 3^{\mathrm{ii}}$ & 0.98 & 2.40 & $3.185(3)$ & 136 \\
$\mathrm{C} 17-\mathrm{H} 17 B \cdots 8^{\mathrm{i}}$ & 0.99 & 2.40 & $3.102(3)$ & 127 \\
$\mathrm{C} 18-\mathrm{H} 18 \cdots \mathrm{F} 10^{\mathrm{iii}}$ & 1.00 & 2.60 & $3.104(3)$ & 111 \\
$\mathrm{C} 20-\mathrm{H} 20 \cdots 9^{\mathrm{i}}$ & 0.95 & 2.55 & $3.466(3)$ & 162 \\
$\mathrm{C} 32-\mathrm{H} 32 C \cdots \mathrm{F}^{\mathrm{iv}}$ & 0.98 & 2.36 & $3.146(3)$ & 137 \\
\hline
\end{tabular}

Symmetry codes: (i) $x-1, y, z$; (ii) $-x,-y+1,-z+1$; (iii) $x-1, y+1$, $z$; (iv) $-x+3,-y,-z$.

with $\mathrm{C} 25-\mathrm{F} 9 \cdots \mathrm{Cg} 3^{\text {vii }}$ interactions involving the $\mathrm{C} 3-\mathrm{C} 8$ benzene ring to form chains along [001] (Fig. 2). C17$\mathrm{H} 17 B \cdots \mathrm{O} 8^{\mathrm{i}}$ and $\mathrm{C} 20-\mathrm{H} 20 \cdots \mathrm{F} 9^{\mathrm{i}}$ hydrogen bonds form chains along the $a$-axis direction, forming a two-dimensional network in the $a b$ plane (Fig. 3 and Table 1). In addition, short intermolecular F1 $\cdots \mathrm{F}^{\mathrm{iii}}$ and $\mathrm{F} 6 \cdots \mathrm{F} 10^{\mathrm{iii}}$ contacts $[2.846(2)$ and $2.794(2) \AA]$ together with $\mathrm{C} 1-\mathrm{H} 1 A \cdots \mathrm{F} 10^{\mathrm{i}}, \quad \mathrm{C} 16-$ $\mathrm{H} 16 C \cdots \mathrm{F} 3^{\mathrm{ii}}, \quad \mathrm{C} 18-\mathrm{H} 18 \cdots \mathrm{F} 10^{\mathrm{iii}}$ and $\mathrm{C} 32-\mathrm{H} 32 C \cdots \mathrm{F} 8^{\mathrm{iv}}$ hydrogen bonds generate a three-dimensional network with molecules stacked along the $a$-axis direction (Fig. 4) and Table 1).

\section{Database survey}

Crystal structures of sulfonamide (Kang et al., 2015; Chen, Wu et al., 2005) and triazolopyrimidine (Chen, $\mathrm{Li}$ et al., 2005) herbicides have been reported previously. Moreover, the crystal structures of compounds with a triazolopyrimidine ring system and a benzene ring in the molecule, ethyl 2-(5,7-

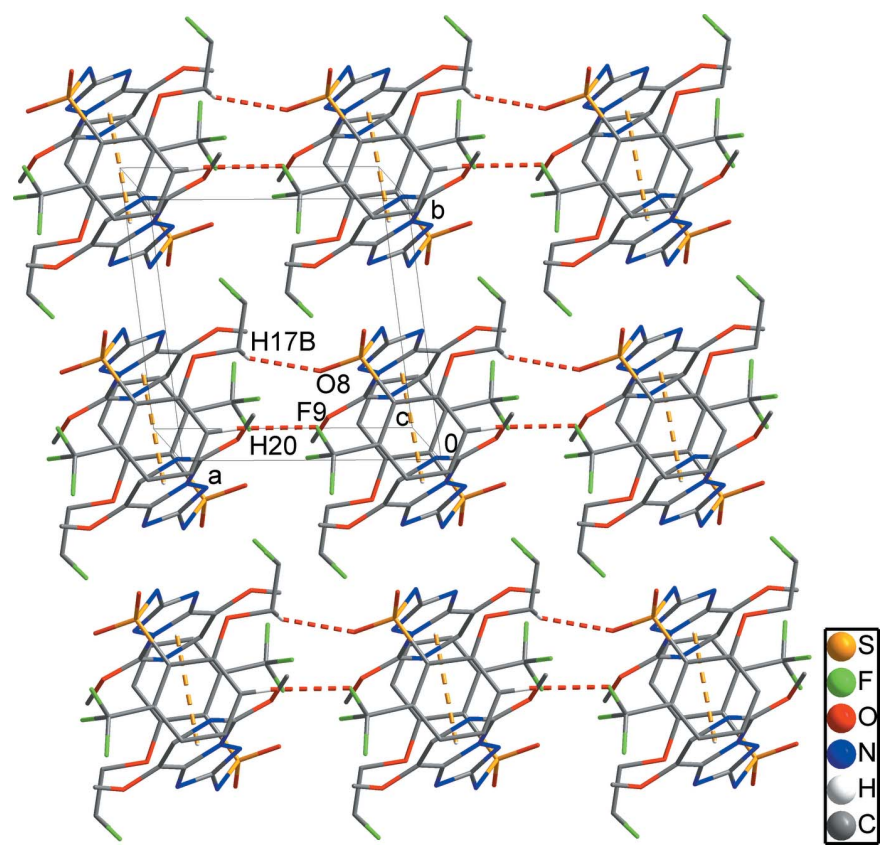

Figure 3

A two-dimensional network in the $a b$ plane. Yellow dashed lines indicate weak intermolecular $\pi-\pi$ interactions. Red dashed lines indicate $\mathrm{C}-$ $\mathrm{F} \cdots \pi$ interactions and $\mathrm{C}-\mathrm{H} \cdots \mathrm{O}$ hydrogen bonds. $\mathrm{H}$ atoms have been omitted for clarity. 


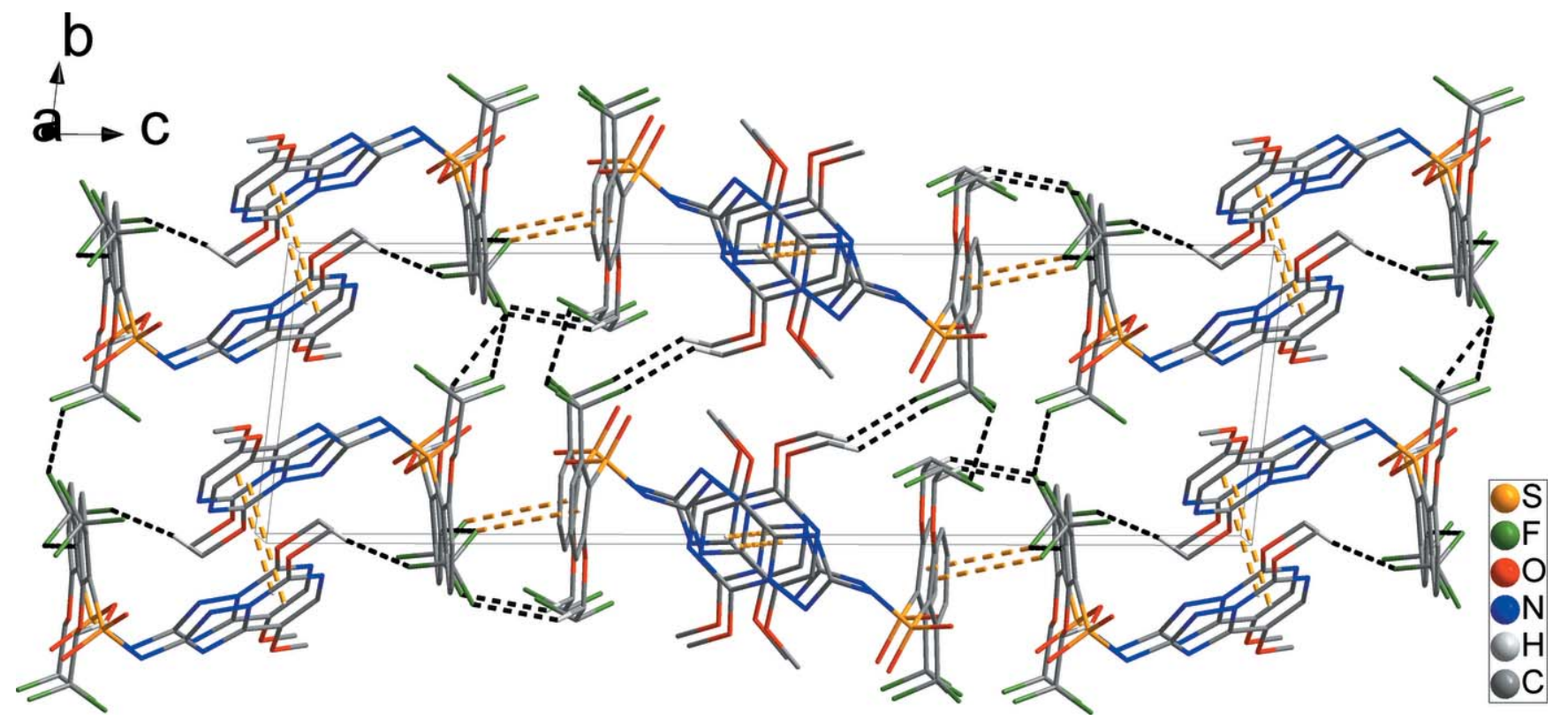

Figure 4

Overall packing showing the three-dimensional network viewed along the $a$-axis direction, $\mathrm{C}-\mathrm{H} \cdots \mathrm{F}$ hydrogen bonds and F $\cdots \mathrm{F}$ intermolecular short contacts are shown as black dashed lines. $\mathrm{H}$ atoms have been omitted for clarity.

dimethyl-1,2,4-triazolo[1,5-a]-pyrimidin-2-yloxy)benzoate (Chen, Li et al., 2005) and 5-(4-Chlorophenoxy)-6-isopropyl-3phenyl-3H-1,2,3-triazolo[4,5-d]-pyrimidin-7(6H)-one (Zeng et al., 2009) have also been reported.

Table 2

Experimental details.

\begin{tabular}{ll}
\hline Crystal data & \\
Chemical formula & $\mathrm{C}_{16} \mathrm{H}_{14} \mathrm{~F}_{5} \mathrm{~N}_{5} \mathrm{O}_{5} \mathrm{~S}$ \\
$M_{\mathrm{r}}$ & 483.38 \\
Crystal system, space group & Triclinic, $P \overline{1}$ \\
Temperature $(\mathrm{K})$ & 173 \\
$a, b, c(\AA)$ & $8.1945(3), 8.3733(3), 28.3277(9)$ \\
$\alpha, \beta, \gamma\left({ }^{\circ}\right)$ & $82.698(2), 84.183(2), 81.814(2)$ \\
$V\left(\AA^{3}\right)$ & $1901.43(12)$ \\
$Z$ & 4 \\
Radiation type & Mo $K \alpha$ \\
$\mu\left(\mathrm{mm}^{-1}\right)$ & 0.26 \\
Crystal size $(\mathrm{mm})$ & $0.40 \times 0.19 \times 0.11$ \\
& \\
Data collection & Bruker APEXII CCD \\
Diffractometer & Multi-scan $(S A D A B S ;$ Bruker, \\
Absorption correction & $2014)$ \\
& $0.646,0.746$ \\
$T_{\min }, T_{\text {max }}$ & $24336,6571,5503$ \\
No. of measured, independent and & \\
$\quad$ observed $[I>2 \sigma(I)]$ reflections & 0.032 \\
$R_{\text {int }}$ & 0.595 \\
$(\text { sin } \theta / \lambda)_{\text {max }}\left(\AA^{-1}\right)$ & \\
Refinement & \\
$R\left[F^{2}>2 \sigma\left(F^{2}\right)\right], w R\left(F^{2}\right), S$ & $0.044,0.106,1.05$ \\
No. of reflections & 6571 \\
No. of parameters & 581 \\
No. of restraints & 1 \\
H-atom treatment & H-atom parameters constrained \\
$\Delta \rho_{\text {max }}, \Delta \rho_{\text {min }}\left(\mathrm{e} \AA^{-3}\right)$ & $0.47,-0.52$ \\
\hline
\end{tabular}

Computer programs: APEX2 and SAINT (Bruker, 2014), SHELXS97 and SHELXTL (Sheldrick, 2008), SHELXL2014 (Sheldrick, 2015), DIAMOND (Brandenburg, 2010) and publCIF (Westrip, 2010).

\section{Synthesis and crystallization}

The title compound was purchased from the Dr. Ehrenstorfer GmbH Company. Single crystals were obtained by slow evaporation of an acetonitrile solution.

\section{Refinement}

Crystal data, data collection and structure refinement details are summarized in Table 2. All $\mathrm{H}$ atoms were positioned geometrically and refined using a riding model with $d(\mathrm{~N}-\mathrm{H})$ $=0.88 \AA, U_{\text {iso }}(\mathrm{H})=1.2 U_{\text {eq }}(\mathrm{C})$ for the $\mathrm{N}-\mathrm{H}$ group, $d(\mathrm{C}-\mathrm{H})=$ $0.95 \AA, U_{\text {iso }}(\mathrm{H})=1.2 U_{\text {eq }}(\mathrm{C})$ for aromatic $\mathrm{C}-\mathrm{H}, d(\mathrm{C}-\mathrm{H})=$ $0.98 \AA, U_{\text {iso }}(\mathrm{H})=1.5 U_{\text {eq }}(\mathrm{C})$ for methyl group, $d(\mathrm{C}-\mathrm{H})=$ $0.99 \AA, U_{\text {iso }}(\mathrm{H})=1.2 U_{\text {eq }}(\mathrm{C})$ for $\mathrm{Csp}^{3}-\mathrm{H}$, and $d(\mathrm{C}-\mathrm{H})=$ $1.00 \AA, U_{\text {iso }}(\mathrm{H})=1.5 U_{\text {eq }}(\mathrm{C})$ for $\mathrm{Csp} p^{3}-\mathrm{H}$.

\section{Funding information}

This research was supported by the Basic Science Program through the National Research Foundation of Korea (NRF) funded by the Ministry of Education, Science and Technology (No. 2015R1D1A4A01020317 and 2017R1D1A3A03000534).

\section{References}

AboulWafa, O. M., Farghaly, A. M., Teleb, M. \& Sinoussy, K. S. (2014). Acta Cryst. E70, o672-o673.

Brandenburg, K. (2010). DIAMOND. Crystal Impact GbR, Bonn, Germany.

Bruker (2014). APEX2, SAINT and SADABS. Bruker AXS Inc., Madison, Wisconsin, USA.

Chen, Q., Li, Y.-X., Hu, X.-W. \& Yang, G.-F. (2005). Acta Cryst. E61, o3842-o3843.

Chen, Q., Li, Y.-X., Hu, X.-W. \& Yang, G.-F. (2005). Acta Cryst. E61, o3842-o3843. 
Jabusch, T. W. \& Tjeerdema, R. S. (2005). J. Agric. Food Chem. 53, 7179-7183.

Kang, G., Kim, J., Jeon, Y. \& Kim, T. H. (2015). Acta Cryst. E71, o429.

Kumar, M., Mallesha, L., Sridhar, M. A., Kapoor, K., Gupta, V. K. \& Kant, R. (2012). Acta Cryst. E68, o2800.

Sheldrick, G. M. (2008). Acta Cryst. A64, 112-122.

Sheldrick, G. M. (2015). Acta Cryst. C71, 3-8.

Westrip, S. P. (2010). J. Appl. Cryst. 43, 920-925.
Willingham, S. D., McCauley, G. N., Senseman, S. A., Chandler, J. M., Richburg, J. S., Lassiter, R. B. \& Mann, R. K. (2008). Weed Technol. 22, 114-118.

Yasuor, H., Osuna, M. D., Ortiz, A., Saldaín, N. E., Eckert, J. W. \& Fischer, A. J. (2009). J. Agric. Food Chem. 57, 3653-3660.

Zeng, X.-H., Liu, X.-L., Deng, S.-H., Chen, P. \& Wang, H.-M. (2009). Acta Cryst. E65, o2583-o2584. 


\section{supporting information}

Acta Cryst. (2017). E73, 1312-1315 [https://doi.org/10.1107/S2056989017011458]

\section{Crystal structure of penoxsulam}

\section{Hyunjin Park, Jineun Kim, Hojae Chiang and Tae Ho Kim}

\section{Computing details}

Data collection: APEX2 (Bruker, 2014); cell refinement: SAINT (Bruker, 2014); data reduction: SAINT (Bruker, 2014); program(s) used to solve structure: SHELXS97 (Sheldrick, 2008); program(s) used to refine structure: SHELXL2014 (Sheldrick, 2015); molecular graphics: DIAMOND (Brandenburg, 2010); software used to prepare material for publication: SHELXTL (Sheldrick, 2008) and publCIF (Westrip, 2010).

2-(2,2-Difluoroethoxy)- $N$-(5,8-dimethoxy-1,2,4-triazolo[1,5-c]pyrimidin-2-

yl)-6-(trifluoromethyl)benzenesulfonamide

Crystal data

$\mathrm{C}_{16} \mathrm{H}_{14} \mathrm{~F}_{5} \mathrm{~N}_{5} \mathrm{O}_{5} \mathrm{~S}$

$M_{r}=483.38$

Triclinic, $P \overline{1}$

$a=8.1945$ (3) $\AA$

$b=8.3733(3) \AA$

$c=28.3277$ (9) $\AA$

$\alpha=82.698(2)^{\circ}$

$\beta=84.183(2)^{\circ}$

$\gamma=81.814(2)^{\circ}$

$V=1901.43(12) \AA^{3}$

Data collection

Bruker APEXII CCD

diffractometer

$\varphi$ and $\omega$ scans

Absorption correction: multi-scan

(SADABS; Bruker, 2014)

$T_{\min }=0.646, T_{\max }=0.746$

24336 measured reflections

\section{Refinement}

Refinement on $F^{2}$

Least-squares matrix: full

$R\left[F^{2}>2 \sigma\left(F^{2}\right)\right]=0.044$

$w R\left(F^{2}\right)=0.106$

$S=1.05$

6571 reflections

581 parameters

1 restraint
$Z=4$

$F(000)=984$

$D_{\mathrm{x}}=1.689 \mathrm{Mg} \mathrm{m}^{-3}$

Mo $K \alpha$ radiation, $\lambda=0.71073 \AA$

Cell parameters from 9990 reflections

$\theta=2.5-27.3^{\circ}$

$\mu=0.26 \mathrm{~mm}^{-1}$

$T=173 \mathrm{~K}$

Plate, colourless

$0.40 \times 0.19 \times 0.11 \mathrm{~mm}$

6571 independent reflections

5503 reflections with $I>2 \sigma(I)$

$R_{\text {int }}=0.032$

$\theta_{\max }=25.0^{\circ}, \theta_{\min }=1.5^{\circ}$

$h=-9 \rightarrow 9$

$k=-9 \rightarrow 9$

$l=-32 \rightarrow 33$

Hydrogen site location: inferred from neighbouring sites

$\mathrm{H}$-atom parameters constrained

$w=1 /\left[\sigma^{2}\left(F_{\mathrm{o}}{ }^{2}\right)+(0.0438 P)^{2}+1.5689 P\right]$

where $P=\left(F_{\mathrm{o}}^{2}+2 F_{\mathrm{c}}{ }^{2}\right) / 3$

$(\Delta / \sigma)_{\max }=0.001$

$\Delta \rho_{\max }=0.47$ e $\AA^{-3}$

$\Delta \rho_{\min }=-0.52$ e $\AA^{-3}$ 


\section{Special details}

Geometry. All esds (except the esd in the dihedral angle between two 1.s. planes) are estimated using the full covariance matrix. The cell esds are taken into account individually in the estimation of esds in distances, angles and torsion angles; correlations between esds in cell parameters are only used when they are defined by crystal symmetry. An approximate (isotropic) treatment of cell esds is used for estimating esds involving l.s. planes.

Fractional atomic coordinates and isotropic or equivalent isotropic displacement parameters $\left(\AA^{2}\right)$

\begin{tabular}{|c|c|c|c|c|}
\hline & $x$ & $y$ & $z$ & $U_{\text {iso }} * / U_{\text {eq }}$ \\
\hline S1 & $0.40491(7)$ & $0.27322(7)$ & $0.33986(2)$ & $0.02327(15)$ \\
\hline $\mathrm{S} 2$ & $1.17808(7)$ & $0.29094(7)$ & $0.15763(2)$ & $0.02404(15)$ \\
\hline F1 & $0.6784(2)$ & $-0.21313(19)$ & $0.28932(6)$ & $0.0473(4)$ \\
\hline $\mathrm{F} 2$ & $0.6798(2)$ & $-0.2261(2)$ & $0.36604(6)$ & $0.0526(5)$ \\
\hline F3 & $0.0164(2)$ & $0.53003(19)$ & $0.34514(6)$ & 0.0477 (4) \\
\hline F4 & $0.18482(19)$ & $0.53143(16)$ & $0.28142(5)$ & $0.0326(3)$ \\
\hline F5 & $-0.0713(2)$ & $0.51888(19)$ & $0.27738(6)$ & 0.0465 (4) \\
\hline F6 & $0.6485(2)$ & $0.5512(2)$ & $0.21727(6)$ & $0.0501(4)$ \\
\hline F7 & $0.6787(3)$ & $0.5868(2)$ & $0.13991(7)$ & $0.0629(5)$ \\
\hline F8 & $1.4342(2)$ & $-0.0947(2)$ & $0.15363(6)$ & $0.0505(5)$ \\
\hline F9 & $1.39010(18)$ & 0.03048 (19) & $0.21686(5)$ & $0.0381(4)$ \\
\hline F10 & $1.3647(2)$ & -0.22070 (19) & $0.22130(6)$ & $0.0523(5)$ \\
\hline $\mathrm{O} 1$ & $0.4016(2)$ & $-0.06137(18)$ & $0.33033(6)$ & $0.0292(4)$ \\
\hline $\mathrm{O} 2$ & $0.3455(2)$ & $0.42426(19)$ & $0.35764(6)$ & $0.0343(4)$ \\
\hline $\mathrm{O} 3$ & $0.5333(2)$ & $0.2632(2)$ & $0.30221(6)$ & $0.0356(4)$ \\
\hline $\mathrm{O} 4$ & $0.3555(3)$ & $-0.3031(3)$ & $0.52168(8)$ & $0.0591(6)$ \\
\hline $\mathrm{O} 5$ & $0.0298(3)$ & $0.3079(3)$ & $0.51945(7)$ & $0.0460(5)$ \\
\hline O6 & $0.8254(2)$ & $0.2966(2)$ & $0.17644(6)$ & $0.0309(4)$ \\
\hline $\mathrm{O} 7$ & $1.1568(2)$ & $0.3944(2)$ & $0.19444(6)$ & $0.0349(4)$ \\
\hline O8 & $1.3393(2)$ & $0.2352(2)$ & $0.13777(6)$ & $0.0334(4)$ \\
\hline O9 & $0.7015(3)$ & $0.3776(3)$ & $-0.02223(8)$ & $0.0535(6)$ \\
\hline $\mathrm{O} 10$ & $1.3171(3)$ & $0.0395(3)$ & $-0.02043(7)$ & $0.0449(5)$ \\
\hline N1 & $0.4795(2)$ & $0.1502(2)$ & $0.38497(7)$ & $0.0250(4)$ \\
\hline $\mathrm{H} 1 \mathrm{~N}$ & 0.5842 & 0.1083 & 0.3822 & $0.030^{*}$ \\
\hline $\mathrm{N} 2$ & $0.4113(3)$ & $-0.0440(2)$ & $0.44874(8)$ & $0.0315(5)$ \\
\hline N3 & $0.2718(3)$ & $0.2139(2)$ & $0.44832(7)$ & $0.0293(5)$ \\
\hline N4 & $0.2201(3)$ & $0.1158(3)$ & $0.48799(7)$ & $0.0360(5)$ \\
\hline N5 & $0.0657(4)$ & $0.0425(4)$ & $0.55905(8)$ & $0.0537(7)$ \\
\hline N6 & $1.0775(3)$ & $0.3946(2)$ & $0.11402(7)$ & $0.0273(5)$ \\
\hline $\mathrm{H} 6 \mathrm{~N}$ & 1.0315 & 0.4945 & 0.1175 & $0.033^{*}$ \\
\hline N7 & $0.9191(3)$ & $0.3767(2)$ & $0.05042(7)$ & $0.0313(5)$ \\
\hline N8 & $1.1790(3)$ & $0.2324(3)$ & $0.05044(7)$ & $0.0303(5)$ \\
\hline N9 & $1.1034(3)$ & $0.2116(3)$ & $0.01107(7)$ & $0.0354(5)$ \\
\hline N10 & $1.0719(3)$ & $0.1088(3)$ & $-0.05969(8)$ & $0.0483(6)$ \\
\hline $\mathrm{C} 1$ & $0.4275(3)$ & $-0.2344(3)$ & $0.33482(10)$ & $0.0319(6)$ \\
\hline H1A & 0.3768 & -0.2749 & 0.3091 & $0.038^{*}$ \\
\hline H1B & 0.3776 & -0.2797 & 0.3661 & $0.038^{*}$ \\
\hline $\mathrm{C} 2$ & $0.6090(4)$ & $-0.2821(3)$ & $0.33091(10)$ & $0.0366(7)$ \\
\hline $\mathrm{H} 2$ & 0.6349 & -0.4030 & 0.3329 & $0.044 *$ \\
\hline
\end{tabular}




\begin{tabular}{|c|c|c|c|c|}
\hline $\mathrm{C} 3$ & $0.2487(3)$ & $0.0195(3)$ & $0.32428(8)$ & $0.0226(5)$ \\
\hline $\mathrm{C} 4$ & 0.1131 (3) & $-0.0560(3)$ & $0.31745(9)$ & $0.0283(6)$ \\
\hline $\mathrm{H} 4$ & 0.1223 & -0.1710 & 0.3198 & $0.034 *$ \\
\hline $\mathrm{C} 5$ & $-0.0334(3)$ & $0.0372(3)$ & $0.30731(9)$ & $0.0319(6)$ \\
\hline H5 & -0.1263 & -0.0142 & 0.3032 & $0.038 *$ \\
\hline C6 & $-0.0480(3)$ & 0.2044 (3) & $0.30301(9)$ & $0.0291(6)$ \\
\hline H6 & -0.1488 & 0.2671 & 0.2944 & $0.035^{*}$ \\
\hline $\mathrm{C} 7$ & 0.0837 (3) & $0.2816(3)$ & $0.31124(8)$ & $0.0219(5)$ \\
\hline $\mathrm{C} 8$ & $0.2322(3)$ & 0.1891 (3) & $0.32403(7)$ & $0.0188(5)$ \\
\hline C9 & $0.0556(3)$ & $0.4652(3)$ & $0.30368(9)$ & $0.0303(6)$ \\
\hline $\mathrm{C} 10$ & $0.3842(3)$ & $0.1093(3)$ & $0.42737(8)$ & $0.0264(5)$ \\
\hline C11 & $0.3046(3)$ & -0.0355 & $0.48755(9)$ & $0.0303(6)$ \\
\hline $\mathrm{C} 12$ & $0.2670(4)$ & $-0.1556(4)$ & $0.52547(11)$ & $0.0451(8)$ \\
\hline $\mathrm{C} 13$ & 0.1508 (4) & $-0.1123(4)$ & $0.55917(10)$ & $0.0472(8)$ \\
\hline H13 & 0.1246 & -0.1919 & 0.5848 & $0.057 *$ \\
\hline C14 & 0.1007 (4) & $0.1546(4)$ & $0.52415(9)$ & $0.0400(7)$ \\
\hline $\mathrm{C} 15$ & $0.3152(5)$ & $-0.4323(4)$ & 0.55754 (13) & $0.0761(13)$ \\
\hline H15A & 0.3362 & -0.4064 & 0.5890 & $0.114^{*}$ \\
\hline H15B & 0.3837 & -0.5338 & 0.5506 & $0.114 *$ \\
\hline $\mathrm{H} 15 \mathrm{C}$ & 0.1981 & -0.4448 & 0.5575 & $0.114^{*}$ \\
\hline C16 & $-0.0932(4)$ & $0.3529(4)$ & $0.55773(11)$ & $0.0618(10)$ \\
\hline H16A & -0.1876 & 0.2927 & 0.5580 & $0.093 *$ \\
\hline H16B & -0.1309 & 0.4697 & 0.5526 & $0.093 *$ \\
\hline $\mathrm{H} 16 \mathrm{C}$ & -0.0443 & 0.3266 & 0.5884 & $0.093 *$ \\
\hline $\mathrm{C} 17$ & $0.6501(3)$ & $0.3241(3)$ & 0.17727 (11) & $0.0388(7)$ \\
\hline H17A & 0.5996 & 0.2640 & 0.2062 & $0.047 *$ \\
\hline H17B & 0.6132 & 0.2872 & 0.1487 & $0.047 *$ \\
\hline C18 & 0.6008 (4) & $0.5018(4)$ & $0.17763(12)$ & $0.0460(8)$ \\
\hline H18 & 0.4782 & 0.5284 & 0.1767 & $0.055^{*}$ \\
\hline C19 & 0.8998 (3) & $0.1400(3)$ & $0.17952(8)$ & $0.0244(5)$ \\
\hline $\mathrm{C} 20$ & $0.8126(3)$ & $0.0072(3)$ & $0.18630(9)$ & $0.0326(6)$ \\
\hline $\mathrm{H} 20$ & 0.6953 & 0.0231 & 0.1871 & $0.039 *$ \\
\hline $\mathrm{C} 21$ & $0.8963(3)$ & -0.1464 & $0.19184(9)$ & $0.0350(6)$ \\
\hline H21 & 0.8369 & -0.2372 & 0.1948 & $0.042 *$ \\
\hline $\mathrm{C} 22$ & $1.0651(3)$ & $-0.1711(3)$ & $0.19321(9)$ & $0.0321(6)$ \\
\hline $\mathrm{H} 22$ & 1.1208 & -0.2783 & 0.1991 & $0.039 *$ \\
\hline $\mathrm{C} 23$ & $1.1547(3)$ & $-0.0404(3)$ & $0.18604(8)$ & $0.0238(5)$ \\
\hline $\mathrm{C} 24$ & $1.0729(3)$ & $0.1177(3)$ & $0.17622(8)$ & $0.0203(5)$ \\
\hline $\mathrm{C} 25$ & $1.3353(3)$ & $-0.0779(3)$ & $0.19369(9)$ & $0.0344(6)$ \\
\hline $\mathrm{C} 26$ & $1.0613(3)$ & $0.3314(3)$ & $0.07141(8)$ & $0.0257(5)$ \\
\hline $\mathrm{C} 27$ & $0.9510(3)$ & 0.2979 (3) & $0.01135(8)$ & $0.0275(6)$ \\
\hline $\mathrm{C} 28$ & $0.8526(4)$ & $0.2892(3)$ & $-0.02617(10)$ & $0.0407(7)$ \\
\hline $\mathrm{C} 29$ & 0.9154 (4) & 0.1956 (4) & $-0.05974(9)$ & $0.0422(7)$ \\
\hline $\mathrm{H} 29$ & 0.8498 & 0.1880 & -0.0850 & $0.051^{*}$ \\
\hline $\mathrm{C} 30$ & $1.1653(4)$ & 0.1154 (3) & $-0.02503(9)$ & $0.0369(7)$ \\
\hline C31 & $0.5908(5)$ & $0.3640(5)$ & $-0.05730(12)$ & $0.0651(10)$ \\
\hline H31A & 0.6383 & 0.4036 & -0.0891 & $0.098^{*}$ \\
\hline H31B & 0.4841 & 0.4291 & -0.0501 & $0.098^{*}$ \\
\hline
\end{tabular}




$\begin{array}{lllll}\mathrm{H} 31 \mathrm{C} & 0.5747 & 0.2500 & -0.0567 & 0.098^{*} \\ \mathrm{C} 32 & 1.3861(4) & -0.0568(4) & -0.05853(11) & 0.0574(9) \\ \mathrm{H} 32 \mathrm{~A} & 1.3238 & -0.1487 & -0.0580 & 0.086^{*} \\ \mathrm{H} 32 \mathrm{~B} & 1.5023 & -0.0976 & -0.0540 & 0.086^{*} \\ \mathrm{H} 32 \mathrm{C} & 1.3789 & 0.0106 & -0.0894 & 0.086^{*}\end{array}$

Atomic displacement parameters $\left(\AA^{2}\right)$

\begin{tabular}{|c|c|c|c|c|c|c|}
\hline & $U^{11}$ & $U^{22}$ & $U^{33}$ & $U^{12}$ & $U^{13}$ & $U^{23}$ \\
\hline $\mathrm{S} 1$ & $0.0248(3)$ & $0.0191(3)$ & $0.0273(3)$ & $-0.0096(2)$ & $-0.0074(2)$ & $0.0035(2)$ \\
\hline $\mathrm{S} 2$ & 0.0227 (3) & $0.0273(3)$ & $0.0243(3)$ & $-0.0104(3)$ & $-0.0056(2)$ & $-0.0001(2)$ \\
\hline F1 & 0.0495 (11) & $0.0363(9)$ & $0.0517(10)$ & $-0.0063(8)$ & $0.0157(8)$ & $-0.0038(8)$ \\
\hline $\mathrm{F} 2$ & $0.0434(11)$ & $0.0549(11)$ & $0.0572(11)$ & $0.0058(8)$ & $-0.0152(9)$ & $-0.0032(9)$ \\
\hline F3 & $0.0608(12)$ & $0.0387(9)$ & $0.0427(9)$ & $0.0094(8)$ & $-0.0013(8)$ & $-0.0212(7)$ \\
\hline F4 & $0.0448(9)$ & $0.0202(7)$ & $0.0334(8)$ & $-0.0065(6)$ & $-0.0109(7)$ & $0.0034(6)$ \\
\hline F5 & $0.0383(10)$ & $0.0389(9)$ & $0.0598(11)$ & $0.0152(7)$ & $-0.0215(8)$ & $-0.0052(8)$ \\
\hline F6 & $0.0374(10)$ & $0.0509(10)$ & 0.0675 (12) & -0.0021 & $-0.0103(8)$ & $-0.0271(9)$ \\
\hline F7 & $0.0708(14)$ & $0.0459(10)$ & $0.0689(13)$ & $0.0095(10)$ & $-0.0230(11)$ & $0.0004(9)$ \\
\hline F8 & $0.0353(10)$ & $0.0617(11)$ & $0.0460(10)$ & $0.0086(8)$ & $0.0167(8)$ & $-0.0063(8)$ \\
\hline F9 & $0.0239(8)$ & $0.0547(10)$ & $0.0349(8)$ & -0.0035 & $-0.0082(6)$ & $0.0007(7)$ \\
\hline F10 & $0.0421(11)$ & $0.0414(9)$ & $0.0616(11)$ & $0.0160(8)$ & $-0.0023(8)$ & $0.0140(8)$ \\
\hline O1 & $0.0291(10)$ & $0.0136(8)$ & $0.0455(10)$ & $-0.0016(7)$ & $-0.0089(8)$ & $-0.0020(7)$ \\
\hline $\mathrm{O} 2$ & $0.0486(12)$ & $0.0161(8)$ & $0.0417(10)$ & $-0.0081(8)$ & $-0.0193(9)$ & $-0.0008(7)$ \\
\hline $\mathrm{O} 3$ & $0.0253(10)$ & 0.0465 (11) & $0.0347(10)$ & $-0.0149(8)$ & $-0.0016(8)$ & $0.0073(8)$ \\
\hline $\mathrm{O} 4$ & $0.0559(15)$ & $0.0501(14)$ & $0.0696(15)$ & $-0.0188(12)$ & $-0.0251(12)$ & $0.0290(11)$ \\
\hline $\mathrm{O} 5$ & 0.0544 (14) & $0.0528(13)$ & $0.0349(11)$ & $-0.0212(11)$ & $0.0097(9)$ & $-0.0158(9)$ \\
\hline O6 & $0.0160(9)$ & $0.0283(9)$ & $0.0490(11)$ & $-0.0019(7)$ & $-0.0057(8)$ & $-0.0056(8)$ \\
\hline O7 & $0.0458(12)$ & $0.0342(10)$ & $0.0298(10)$ & $-0.0159(9)$ & $-0.0095(8)$ & $-0.0062(8)$ \\
\hline O8 & $0.0191(9)$ & $0.0468(11)$ & $0.0346(10)$ & $-0.0121(8)$ & $-0.0033(7)$ & $0.0041(8)$ \\
\hline O9 & $0.0629(16)$ & $0.0460(12)$ & $0.0560(13)$ & $-0.0108(11)$ & $-0.0342(11)$ & $0.0043(10)$ \\
\hline $\mathrm{O} 10$ & 0.0409 (13) & $0.0634(13)$ & $0.0343(11)$ & $-0.0178(11)$ & $0.0065(9)$ & $-0.0158(10)$ \\
\hline N1 & $0.0177(11)$ & $0.0273(11)$ & $0.0302(11)$ & $-0.0045(8)$ & $-0.0094(8)$ & $0.0041(9)$ \\
\hline N2 & $0.0320(13)$ & $0.0284(11)$ & $0.0357(12)$ & $-0.0117(10)$ & $-0.0146(10)$ & $0.0086(9)$ \\
\hline N3 & $0.0346(13)$ & $0.0310(11)$ & $0.0249(11)$ & $-0.0144(10)$ & $-0.0046(9)$ & $-0.0004(9)$ \\
\hline N4 & 0.0387 (14) & $0.0455(14)$ & $0.0273(12)$ & $-0.0188(11)$ & $-0.0086(10)$ & $0.0014(10)$ \\
\hline N5 & 0.0623 (19) & $0.079(2)$ & $0.0285(13)$ & $-0.0423(16)$ & $-0.0065(12)$ & 0.0003 (13) \\
\hline N6 & 0.0355 (13) & $0.0201(10)$ & $0.0276(11)$ & $-0.0067(9)$ & $-0.0086(9)$ & $0.0003(8)$ \\
\hline N7 & $0.0346(13)$ & $0.0272(11)$ & $0.0332(12)$ & $-0.0091(10)$ & $-0.0120(10)$ & $0.0055(9)$ \\
\hline N8 & 0.0332 (13) & $0.0358(12)$ & $0.0242(11)$ & $-0.0126(10)$ & $-0.0043(9)$ & $-0.0018(9)$ \\
\hline N9 & $0.0446(15)$ & $0.0384(13)$ & $0.0262(12)$ & $-0.0190(11)$ & $-0.0053(10)$ & $0.0026(10)$ \\
\hline N10 & $0.0610(15)$ & $0.0614(17)$ & $0.0280(12)$ & $-0.0318(12)$ & $-0.0021(12)$ & $-0.0012(11)$ \\
\hline $\mathrm{C} 1$ & $0.0404(16)$ & $0.0120(11)$ & $0.0417(15)$ & $-0.0024(11)$ & $-0.0001(12)$ & $-0.0003(10)$ \\
\hline $\mathrm{C} 2$ & 0.0409 (17) & $0.0201(13)$ & $0.0451(17)$ & $0.0000(12)$ & 0.0027 (13) & $0.0004(12)$ \\
\hline $\mathrm{C} 3$ & 0.0243 (14) & $0.0218(12)$ & $0.0212(12)$ & $-0.0040(10)$ & $-0.0013(10)$ & $0.0005(9)$ \\
\hline $\mathrm{C} 4$ & $0.0325(15)$ & $0.0232(12)$ & 0.0317 (14) & $-0.0128(11)$ & $-0.0013(11)$ & $-0.0039(10)$ \\
\hline $\mathrm{C} 5$ & 0.0253 (14) & $0.0408(15)$ & $0.0337(14)$ & $-0.0175(12)$ & $0.0000(11)$ & $-0.0069(12)$ \\
\hline C6 & 0.0192 (13) & $0.0387(15)$ & $0.0300(13)$ & $-0.0029(11)$ & $-0.0016(10)$ & $-0.0075(11)$ \\
\hline $\mathrm{C} 7$ & $0.0218(13)$ & 0.0239 (12) & $0.0199(11)$ & $-0.0014(10)$ & $-0.0008(9)$ & $-0.0049(9)$ \\
\hline
\end{tabular}




\begin{tabular}{lllllll} 
C8 & $0.0214(13)$ & $0.0177(11)$ & $0.0180(11)$ & $-0.0064(9)$ & $-0.0005(9)$ & $-0.0011(9)$ \\
C9 & $0.0319(15)$ & $0.0264(13)$ & $0.0319(14)$ & $0.0070(11)$ & $-0.0075(11)$ & $-0.0090(11)$ \\
C10 & $0.0281(14)$ & $0.0276(13)$ & $0.0268(13)$ & $-0.0126(11)$ & $-0.0119(11)$ & $0.0021(10)$ \\
C11 & $0.0300(15)$ & $0.0359(15)$ & $0.0285(14)$ & $-0.0172(12)$ & $-0.0143(11)$ & $0.0068(11)$ \\
C12 & $0.0468(19)$ & $0.0523(19)$ & $0.0403(17)$ & $-0.0251(16)$ & $-0.0251(15)$ & $0.0175(14)$ \\
C13 & $0.056(2)$ & $0.065(2)$ & $0.0266(15)$ & $-0.0373(18)$ & $-0.0174(14)$ & $0.0175(14)$ \\
C14 & $0.0433(18)$ & $0.059(2)$ & $0.0240(14)$ & $-0.0256(15)$ & $-0.0038(12)$ & $-0.0066(13)$ \\
C15 & $0.099(3)$ & $0.062(2)$ & $0.072(2)$ & $-0.043(2)$ & $-0.039(2)$ & $0.0379(19)$ \\
C16 & $0.071(2)$ & $0.078(2)$ & $0.0443(19)$ & $-0.036(2)$ & $0.0250(17)$ & $-0.0330(18)$ \\
C17 & $0.0167(14)$ & $0.0439(16)$ & $0.0591(18)$ & $-0.0001(12)$ & $-0.0096(12)$ & $-0.0178(14)$ \\
C18 & $0.0244(16)$ & $0.0511(18)$ & $0.067(2)$ & $0.0050(14)$ & $-0.0176(14)$ & $-0.0227(16)$ \\
C19 & $0.0220(13)$ & $0.0267(13)$ & $0.0252(12)$ & $-0.0033(10)$ & $-0.0033(10)$ & $-0.0045(10)$ \\
C20 & $0.0242(14)$ & $0.0402(15)$ & $0.0362(15)$ & $-0.0136(12)$ & $-0.0006(11)$ & $-0.0065(12)$ \\
C21 & $0.0431(18)$ & $0.0301(14)$ & $0.0355(15)$ & $-0.0191(13)$ & $0.0012(12)$ & $-0.0059(11)$ \\
C22 & $0.0437(17)$ & $0.0238(13)$ & $0.0280(13)$ & $-0.0046(12)$ & $0.0018(12)$ & $-0.0036(10)$ \\
C23 & $0.0251(13)$ & $0.0263(13)$ & $0.0185(11)$ & $-0.0025(10)$ & $0.0038(10)$ & $-0.0020(10)$ \\
C24 & $0.0182(12)$ & $0.0238(12)$ & $0.0195(11)$ & $-0.0059(10)$ & $-0.0009(9)$ & $-0.0022(9)$ \\
C25 & $0.0301(15)$ & $0.0367(15)$ & $0.0312(14)$ & $0.0040(12)$ & $0.0026(12)$ & $0.0019(12)$ \\
C26 & $0.0300(14)$ & $0.0230(12)$ & $0.0254(13)$ & $-0.0114(11)$ & $-0.0061(11)$ & $0.0043(10)$ \\
C27 & $0.0373(16)$ & $0.0241(13)$ & $0.0230(13)$ & $-0.0144(11)$ & $-0.0087(11)$ & $0.0072(10)$ \\
C28 & $0.053(2)$ & $0.0354(15)$ & $0.0367(16)$ & $-0.0185(14)$ & $-0.0211(14)$ & $0.0123(13)$ \\
C29 & $0.0613(17)$ & $0.0504(18)$ & $0.0212(13)$ & $-0.0282(13)$ & $-0.0155(13)$ & $0.0046(12)$ \\
C30 & $0.0489(19)$ & $0.0424(16)$ & $0.0233(14)$ & $-0.0242(14)$ & $0.0030(12)$ & $-0.0027(12)$ \\
C31 & $0.066(3)$ & $0.073(2)$ & $0.062(2)$ & $-0.022(2)$ & $-0.0397(19)$ & $0.0113(18)$ \\
C32 & $0.056(2)$ & $0.081(2)$ & $0.0413(18)$ & $-0.0272(19)$ & $0.0199(16)$ & $-0.0260(17)$ \\
& & & & & & \\
\hline
\end{tabular}

Geometric parameters $\left(A,{ }^{o}\right)$

\begin{tabular}{llll}
\hline $\mathrm{S} 1-\mathrm{O} 3$ & $1.4217(18)$ & $\mathrm{N} 10-\mathrm{C} 30$ & $1.316(4)$ \\
$\mathrm{S} 1-\mathrm{O} 2$ & $1.4238(18)$ & $\mathrm{N} 10-\mathrm{C} 29$ & $1.381(4)$ \\
$\mathrm{S} 1-\mathrm{N} 1$ & $1.6466(19)$ & $\mathrm{C} 1-\mathrm{C} 2$ & $1.481(4)$ \\
$\mathrm{S} 1-\mathrm{C} 8$ & $1.787(2)$ & $\mathrm{C} 1-\mathrm{H} 1 \mathrm{~A}$ & 0.9900 \\
$\mathrm{~S} 2-\mathrm{O} 7$ & $1.4207(18)$ & $\mathrm{C} 1-\mathrm{H} 1 \mathrm{~B}$ & 0.9900 \\
$\mathrm{~S} 2-\mathrm{O} 8$ & $1.4210(18)$ & $\mathrm{C} 2-\mathrm{H} 2$ & 1.0000 \\
$\mathrm{~S} 2-\mathrm{N} 6$ & $1.640(2)$ & $\mathrm{C} 3-\mathrm{C} 4$ & $1.395(3)$ \\
$\mathrm{S} 2-\mathrm{C} 24$ & $1.788(2)$ & $\mathrm{C} 3-\mathrm{C} 8$ & $1.406(3)$ \\
$\mathrm{F} 1-\mathrm{C} 2$ & $1.353(3)$ & $\mathrm{C} 4-\mathrm{C} 5$ & $1.371(4)$ \\
$\mathrm{F} 2-\mathrm{C} 2$ & $1.364(3)$ & $\mathrm{C} 4-\mathrm{H} 4$ & 0.9500 \\
F3-C9 & $1.346(3)$ & $\mathrm{C} 5-\mathrm{C} 6$ & $1.379(4)$ \\
$\mathrm{F} 4-\mathrm{C} 9$ & $1.330(3)$ & $\mathrm{C} 5-\mathrm{H} 5$ & 0.9500 \\
$\mathrm{~F} 5-\mathrm{C} 9$ & $1.333(3)$ & $\mathrm{C} 6-\mathrm{C} 7$ & $1.388(3)$ \\
$\mathrm{F} 6-\mathrm{C} 18$ & $1.355(3)$ & $\mathrm{C} 6-\mathrm{H} 6$ & 0.9500 \\
$\mathrm{~F} 7-\mathrm{C} 18$ & $1.361(4)$ & $\mathrm{C} 7-\mathrm{C} 8$ & $1.401(3)$ \\
F8-C25 & $1.336(3)$ & $\mathrm{C} 7-\mathrm{C} 9$ & $1.513(3)$ \\
$\mathrm{F} 9-\mathrm{C} 25$ & $1.335(3)$ & $\mathrm{C} 11-\mathrm{C} 12$ & $1.417(4)$ \\
$\mathrm{F} 10-\mathrm{C} 25$ & $1.348(3)$ & $\mathrm{C} 12-\mathrm{C} 13$ & 0.9500 \\
$\mathrm{O} 1-\mathrm{C} 3$ & $1.353(3)$ & $\mathrm{C} 13-\mathrm{H} 13$ & 0.9800 \\
$\mathrm{O} 1-\mathrm{C} 1$ & $1.425(3)$ & $\mathrm{C} 15-\mathrm{H} 15 \mathrm{~A}$ &
\end{tabular}




\begin{tabular}{|c|c|c|c|}
\hline $\mathrm{O} 4-\mathrm{C} 12$ & $1.351(4)$ & C15-H15B & 0.9800 \\
\hline $\mathrm{O} 4-\mathrm{C} 15$ & $1.438(4)$ & $\mathrm{C} 15-\mathrm{H} 15 \mathrm{C}$ & 0.9800 \\
\hline $\mathrm{O} 5-\mathrm{C} 14$ & $1.328(4)$ & $\mathrm{C} 16-\mathrm{H} 16 \mathrm{~A}$ & 0.9800 \\
\hline $\mathrm{O} 5-\mathrm{C} 16$ & $1.452(3)$ & C16-H16B & 0.9800 \\
\hline $\mathrm{O} 6-\mathrm{C} 19$ & $1.361(3)$ & $\mathrm{C} 16-\mathrm{H} 16 \mathrm{C}$ & 0.9800 \\
\hline $\mathrm{O} 6-\mathrm{C} 17$ & $1.420(3)$ & $\mathrm{C} 17-\mathrm{C} 18$ & $1.487(4)$ \\
\hline $\mathrm{O} 9-\mathrm{C} 28$ & $1.351(4)$ & C17-H17A & 0.9900 \\
\hline $\mathrm{O} 9-\mathrm{C} 31$ & $1.436(4)$ & C17-H17B & 0.9900 \\
\hline $\mathrm{O} 10-\mathrm{C} 30$ & $1.324(4)$ & C18-H18 & 1.0000 \\
\hline $\mathrm{O} 10-\mathrm{C} 32$ & $1.450(3)$ & $\mathrm{C} 19-\mathrm{C} 20$ & $1.389(3)$ \\
\hline $\mathrm{N} 1-\mathrm{C} 10$ & $1.397(3)$ & $\mathrm{C} 19-\mathrm{C} 24$ & $1.399(3)$ \\
\hline $\mathrm{N} 1-\mathrm{H} 1 \mathrm{~N}$ & 0.8800 & $\mathrm{C} 20-\mathrm{C} 21$ & $1.367(4)$ \\
\hline $\mathrm{N} 2-\mathrm{C} 11$ & $1.336(3)$ & $\mathrm{C} 20-\mathrm{H} 20$ & 0.9500 \\
\hline $\mathrm{N} 2-\mathrm{C} 10$ & $1.346(3)$ & $\mathrm{C} 21-\mathrm{C} 22$ & $1.373(4)$ \\
\hline $\mathrm{N} 3-\mathrm{C} 10$ & $1.326(3)$ & $\mathrm{C} 21-\mathrm{H} 21$ & 0.9500 \\
\hline $\mathrm{N} 3-\mathrm{N} 4$ & $1.373(3)$ & $\mathrm{C} 22-\mathrm{C} 23$ & $1.386(3)$ \\
\hline $\mathrm{N} 4-\mathrm{C} 11$ & $1.355(3)$ & $\mathrm{C} 22-\mathrm{H} 22$ & 0.9500 \\
\hline $\mathrm{N} 4-\mathrm{C} 14$ & $1.380(4)$ & $\mathrm{C} 23-\mathrm{C} 24$ & $1.403(3)$ \\
\hline N5-C14 & $1.313(4)$ & $\mathrm{C} 23-\mathrm{C} 25$ & $1.501(4)$ \\
\hline N5-C13 & $1.382(4)$ & $\mathrm{C} 27-\mathrm{C} 28$ & $1.413(4)$ \\
\hline N6-C26 & $1.403(3)$ & $\mathrm{C} 28-\mathrm{C} 29$ & $1.326(4)$ \\
\hline N6-H6N & 0.8800 & $\mathrm{C} 29-\mathrm{H} 29$ & 0.9500 \\
\hline N7-C27 & $1.344(3)$ & $\mathrm{C} 31-\mathrm{H} 31 \mathrm{~A}$ & 0.9800 \\
\hline $\mathrm{N} 7-\mathrm{C} 26$ & $1.346(3)$ & $\mathrm{C} 31-\mathrm{H} 31 \mathrm{~B}$ & 0.9800 \\
\hline $\mathrm{N} 8-\mathrm{C} 26$ & $1.323(3)$ & $\mathrm{C} 31-\mathrm{H} 31 \mathrm{C}$ & 0.9800 \\
\hline N8-N9 & $1.370(3)$ & $\mathrm{C} 32-\mathrm{H} 32 \mathrm{~A}$ & 0.9800 \\
\hline N9- $\mathrm{C} 27$ & $1.350(3)$ & $\mathrm{C} 32-\mathrm{H} 32 \mathrm{~B}$ & 0.9800 \\
\hline N9- $\mathrm{C} 30$ & $1.393(3)$ & $\mathrm{C} 32-\mathrm{H} 32 \mathrm{C}$ & 0.9800 \\
\hline $\mathrm{O} 3-\mathrm{S} 1-\mathrm{O} 2$ & $120.60(11)$ & $\mathrm{C} 12-\mathrm{C} 13-\mathrm{N} 5$ & $123.4(3)$ \\
\hline $\mathrm{O} 3-\mathrm{S} 1-\mathrm{N} 1$ & $104.84(11)$ & $\mathrm{C} 12-\mathrm{C} 13-\mathrm{H} 13$ & 118.3 \\
\hline $\mathrm{O} 2-\mathrm{S} 1-\mathrm{N} 1$ & $106.54(10)$ & $\mathrm{N} 5-\mathrm{C} 13-\mathrm{H} 13$ & 118.3 \\
\hline $\mathrm{O} 3-\mathrm{S} 1-\mathrm{C} 8$ & $109.19(10)$ & $\mathrm{N} 5-\mathrm{C} 14-\mathrm{O} 5$ & $125.9(3)$ \\
\hline $\mathrm{O} 2-\mathrm{S} 1-\mathrm{C} 8$ & $108.35(11)$ & $\mathrm{N} 5-\mathrm{C} 14-\mathrm{N} 4$ & $119.6(3)$ \\
\hline $\mathrm{N} 1-\mathrm{S} 1-\mathrm{C} 8$ & $106.43(10)$ & $\mathrm{O} 5-\mathrm{C} 14-\mathrm{N} 4$ & $114.5(2)$ \\
\hline $\mathrm{O} 7-\mathrm{S} 2-\mathrm{O} 8$ & $120.38(11)$ & $\mathrm{O} 4-\mathrm{C} 15-\mathrm{H} 15 \mathrm{~A}$ & 109.5 \\
\hline $\mathrm{O} 7-\mathrm{S} 2-\mathrm{N} 6$ & $104.97(11)$ & $\mathrm{O} 4-\mathrm{C} 15-\mathrm{H} 15 \mathrm{~B}$ & 109.5 \\
\hline $\mathrm{O} 8-\mathrm{S} 2-\mathrm{N} 6$ & $107.16(11)$ & $\mathrm{H} 15 \mathrm{~A}-\mathrm{C} 15-\mathrm{H} 15 \mathrm{~B}$ & 109.5 \\
\hline $\mathrm{O} 7-\mathrm{S} 2-\mathrm{C} 24$ & $109.88(10)$ & $\mathrm{O} 4-\mathrm{C} 15-\mathrm{H} 15 \mathrm{C}$ & 109.5 \\
\hline $\mathrm{O} 8-\mathrm{S} 2-\mathrm{C} 24$ & $107.92(11)$ & $\mathrm{H} 15 \mathrm{~A}-\mathrm{C} 15-\mathrm{H} 15 \mathrm{C}$ & 109.5 \\
\hline $\mathrm{N} 6-\mathrm{S} 2-\mathrm{C} 24$ & $105.54(10)$ & $\mathrm{H} 15 \mathrm{~B}-\mathrm{C} 15-\mathrm{H} 15 \mathrm{C}$ & 109.5 \\
\hline $\mathrm{C} 3-\mathrm{O} 1-\mathrm{C} 1$ & $120.10(19)$ & $\mathrm{O} 5-\mathrm{C} 16-\mathrm{H} 16 \mathrm{~A}$ & 109.5 \\
\hline $\mathrm{C} 12-\mathrm{O} 4-\mathrm{C} 15$ & $116.5(3)$ & $\mathrm{O} 5-\mathrm{C} 16-\mathrm{H} 16 \mathrm{~B}$ & 109.5 \\
\hline $\mathrm{C} 14-\mathrm{O} 5-\mathrm{C} 16$ & $115.5(2)$ & $\mathrm{H} 16 \mathrm{~A}-\mathrm{C} 16-\mathrm{H} 16 \mathrm{~B}$ & 109.5 \\
\hline $\mathrm{C} 19-\mathrm{O} 6-\mathrm{C} 17$ & $117.77(19)$ & $\mathrm{O} 5-\mathrm{C} 16-\mathrm{H} 16 \mathrm{C}$ & 109.5 \\
\hline $\mathrm{C} 28-\mathrm{O} 9-\mathrm{C} 31$ & $116.7(3)$ & $\mathrm{H} 16 \mathrm{~A}-\mathrm{C} 16-\mathrm{H} 16 \mathrm{C}$ & 109.5 \\
\hline $\mathrm{C} 30-\mathrm{O} 10-\mathrm{C} 32$ & $115.5(2)$ & $\mathrm{H} 16 \mathrm{~B}-\mathrm{C} 16-\mathrm{H} 16 \mathrm{C}$ & 109.5 \\
\hline $\mathrm{C} 10-\mathrm{N} 1-\mathrm{S} 1$ & $123.11(17)$ & $\mathrm{O} 6-\mathrm{C} 17-\mathrm{C} 18$ & $106.7(2)$ \\
\hline
\end{tabular}




\begin{tabular}{|c|c|c|c|}
\hline $\mathrm{C} 10-\mathrm{N} 1-\mathrm{H} 1 \mathrm{~N}$ & 118.4 & $\mathrm{O} 6-\mathrm{C} 17-\mathrm{H} 17 \mathrm{~A}$ & 110.4 \\
\hline $\mathrm{S} 1-\mathrm{N} 1-\mathrm{H} 1 \mathrm{~N}$ & 118.4 & $\mathrm{C} 18-\mathrm{C} 17-\mathrm{H} 17 \mathrm{~A}$ & 110.4 \\
\hline $\mathrm{C} 11-\mathrm{N} 2-\mathrm{C} 10$ & $100.9(2)$ & $\mathrm{O} 6-\mathrm{C} 17-\mathrm{H} 17 \mathrm{~B}$ & 110.4 \\
\hline $\mathrm{C} 10-\mathrm{N} 3-\mathrm{N} 4$ & $100.3(2)$ & $\mathrm{C} 18-\mathrm{C} 17-\mathrm{H} 17 \mathrm{~B}$ & 110.4 \\
\hline $\mathrm{C} 11-\mathrm{N} 4-\mathrm{N} 3$ & $109.7(2)$ & $\mathrm{H} 17 \mathrm{~A}-\mathrm{C} 17-\mathrm{H} 17 \mathrm{~B}$ & 108.6 \\
\hline $\mathrm{C} 11-\mathrm{N} 4-\mathrm{C} 14$ & $121.8(2)$ & $\mathrm{F} 6-\mathrm{C} 18-\mathrm{F} 7$ & $105.9(2)$ \\
\hline $\mathrm{N} 3-\mathrm{N} 4-\mathrm{C} 14$ & $128.4(2)$ & $\mathrm{F} 6-\mathrm{C} 18-\mathrm{C} 17$ & $110.2(3)$ \\
\hline $\mathrm{C} 14-\mathrm{N} 5-\mathrm{C} 13$ & $119.3(3)$ & $\mathrm{F} 7-\mathrm{C} 18-\mathrm{C} 17$ & $111.1(2)$ \\
\hline $\mathrm{C} 26-\mathrm{N} 6-\mathrm{S} 2$ & $123.03(17)$ & $\mathrm{F} 6-\mathrm{C} 18-\mathrm{H} 18$ & 109.9 \\
\hline $\mathrm{C} 26-\mathrm{N} 6-\mathrm{H} 6 \mathrm{~N}$ & 118.5 & $\mathrm{~F} 7-\mathrm{C} 18-\mathrm{H} 18$ & 109.9 \\
\hline $\mathrm{S} 2-\mathrm{N} 6-\mathrm{H} 6 \mathrm{~N}$ & 118.5 & $\mathrm{C} 17-\mathrm{C} 18-\mathrm{H} 18$ & 109.9 \\
\hline $\mathrm{C} 27-\mathrm{N} 7-\mathrm{C} 26$ & $100.8(2)$ & $\mathrm{O} 6-\mathrm{C} 19-\mathrm{C} 20$ & $123.2(2)$ \\
\hline $\mathrm{C} 26-\mathrm{N} 8-\mathrm{N} 9$ & $100.1(2)$ & $\mathrm{O} 6-\mathrm{C} 19-\mathrm{C} 24$ & $116.2(2)$ \\
\hline $\mathrm{C} 27-\mathrm{N} 9-\mathrm{N} 8$ & $110.4(2)$ & $\mathrm{C} 20-\mathrm{C} 19-\mathrm{C} 24$ & $120.5(2)$ \\
\hline $\mathrm{C} 27-\mathrm{N} 9-\mathrm{C} 30$ & $121.9(2)$ & $\mathrm{C} 21-\mathrm{C} 20-\mathrm{C} 19$ & $119.7(2)$ \\
\hline $\mathrm{N} 8-\mathrm{N} 9-\mathrm{C} 30$ & $127.7(2)$ & $\mathrm{C} 21-\mathrm{C} 20-\mathrm{H} 20$ & 120.1 \\
\hline $\mathrm{C} 30-\mathrm{N} 10-\mathrm{C} 29$ & $120.1(3)$ & $\mathrm{C} 19-\mathrm{C} 20-\mathrm{H} 20$ & 120.1 \\
\hline $\mathrm{O} 1-\mathrm{C} 1-\mathrm{C} 2$ & $106.3(2)$ & $\mathrm{C} 20-\mathrm{C} 21-\mathrm{C} 22$ & $120.8(2)$ \\
\hline $\mathrm{O} 1-\mathrm{C} 1-\mathrm{H} 1 \mathrm{~A}$ & 110.5 & $\mathrm{C} 20-\mathrm{C} 21-\mathrm{H} 21$ & 119.6 \\
\hline $\mathrm{C} 2-\mathrm{C} 1-\mathrm{H} 1 \mathrm{~A}$ & 110.5 & $\mathrm{C} 22-\mathrm{C} 21-\mathrm{H} 21$ & 119.6 \\
\hline $\mathrm{O} 1-\mathrm{C} 1-\mathrm{H} 1 \mathrm{~B}$ & 110.5 & $\mathrm{C} 21-\mathrm{C} 22-\mathrm{C} 23$ & $120.3(2)$ \\
\hline $\mathrm{C} 2-\mathrm{C} 1-\mathrm{H} 1 \mathrm{~B}$ & 110.5 & $\mathrm{C} 21-\mathrm{C} 22-\mathrm{H} 22$ & 119.8 \\
\hline $\mathrm{H} 1 \mathrm{~A}-\mathrm{C} 1-\mathrm{H} 1 \mathrm{~B}$ & 108.7 & $\mathrm{C} 23-\mathrm{C} 22-\mathrm{H} 22$ & 119.8 \\
\hline $\mathrm{F} 1-\mathrm{C} 2-\mathrm{F} 2$ & $105.6(2)$ & $\mathrm{C} 22-\mathrm{C} 23-\mathrm{C} 24$ & $119.8(2)$ \\
\hline $\mathrm{F} 1-\mathrm{C} 2-\mathrm{C} 1$ & $110.6(2)$ & $\mathrm{C} 22-\mathrm{C} 23-\mathrm{C} 25$ & $116.3(2)$ \\
\hline $\mathrm{F} 2-\mathrm{C} 2-\mathrm{C} 1$ & $110.8(2)$ & $\mathrm{C} 24-\mathrm{C} 23-\mathrm{C} 25$ & $123.7(2)$ \\
\hline $\mathrm{F} 1-\mathrm{C} 2-\mathrm{H} 2$ & 109.9 & $\mathrm{C} 19-\mathrm{C} 24-\mathrm{C} 23$ & $118.3(2)$ \\
\hline $\mathrm{F} 2-\mathrm{C} 2-\mathrm{H} 2$ & 109.9 & $\mathrm{C} 19-\mathrm{C} 24-\mathrm{S} 2$ & $118.21(17)$ \\
\hline $\mathrm{C} 1-\mathrm{C} 2-\mathrm{H} 2$ & 109.9 & $\mathrm{C} 23-\mathrm{C} 24-\mathrm{S} 2$ & $123.49(18)$ \\
\hline $\mathrm{O} 1-\mathrm{C} 3-\mathrm{C} 4$ & $123.6(2)$ & $\mathrm{F} 9-\mathrm{C} 25-\mathrm{F} 8$ & $108.1(2)$ \\
\hline $\mathrm{O} 1-\mathrm{C} 3-\mathrm{C} 8$ & $116.1(2)$ & $\mathrm{F} 9-\mathrm{C} 25-\mathrm{F} 10$ & $105.0(2)$ \\
\hline $\mathrm{C} 4-\mathrm{C} 3-\mathrm{C} 8$ & $120.3(2)$ & $\mathrm{F} 8-\mathrm{C} 25-\mathrm{F} 10$ & $105.2(2)$ \\
\hline $\mathrm{C} 5-\mathrm{C} 4-\mathrm{C} 3$ & $119.5(2)$ & $\mathrm{F} 9-\mathrm{C} 25-\mathrm{C} 23$ & $113.5(2)$ \\
\hline $\mathrm{C} 5-\mathrm{C} 4-\mathrm{H} 4$ & 120.3 & $\mathrm{~F} 8-\mathrm{C} 25-\mathrm{C} 23$ & $114.2(2)$ \\
\hline $\mathrm{C} 3-\mathrm{C} 4-\mathrm{H} 4$ & 120.3 & $\mathrm{~F} 10-\mathrm{C} 25-\mathrm{C} 23$ & $110.2(2)$ \\
\hline $\mathrm{C} 4-\mathrm{C} 5-\mathrm{C} 6$ & $121.0(2)$ & $\mathrm{N} 8-\mathrm{C} 26-\mathrm{N} 7$ & $118.5(2)$ \\
\hline $\mathrm{C} 4-\mathrm{C} 5-\mathrm{H} 5$ & 119.5 & $\mathrm{~N} 8-\mathrm{C} 26-\mathrm{N} 6$ & $123.6(2)$ \\
\hline $\mathrm{C} 6-\mathrm{C} 5-\mathrm{H} 5$ & 119.5 & $\mathrm{~N} 7-\mathrm{C} 26-\mathrm{N} 6$ & $117.9(2)$ \\
\hline $\mathrm{C} 5-\mathrm{C} 6-\mathrm{C} 7$ & $120.4(2)$ & N7-C27-N9 & $110.3(2)$ \\
\hline $\mathrm{C} 5-\mathrm{C} 6-\mathrm{H} 6$ & 119.8 & $\mathrm{~N} 7-\mathrm{C} 27-\mathrm{C} 28$ & $131.1(3)$ \\
\hline $\mathrm{C} 7-\mathrm{C} 6-\mathrm{H} 6$ & 119.8 & N9- $\mathrm{C} 27-\mathrm{C} 28$ & $118.6(2)$ \\
\hline $\mathrm{C} 6-\mathrm{C} 7-\mathrm{C} 8$ & $119.8(2)$ & $\mathrm{C} 29-\mathrm{C} 28-\mathrm{O} 9$ & $128.1(3)$ \\
\hline $\mathrm{C} 6-\mathrm{C} 7-\mathrm{C} 9$ & $115.7(2)$ & $\mathrm{C} 29-\mathrm{C} 28-\mathrm{C} 27$ & $117.8(3)$ \\
\hline $\mathrm{C} 8-\mathrm{C} 7-\mathrm{C} 9$ & $124.5(2)$ & $\mathrm{O} 9-\mathrm{C} 28-\mathrm{C} 27$ & $114.0(3)$ \\
\hline $\mathrm{C} 7-\mathrm{C} 8-\mathrm{C} 3$ & $118.7(2)$ & $\mathrm{C} 28-\mathrm{C} 29-\mathrm{N} 10$ & $122.9(3)$ \\
\hline $\mathrm{C} 7-\mathrm{C} 8-\mathrm{S} 1$ & $124.01(17)$ & $\mathrm{C} 28-\mathrm{C} 29-\mathrm{H} 29$ & 118.5 \\
\hline $\mathrm{C} 3-\mathrm{C} 8-\mathrm{S} 1$ & $117.28(18)$ & $\mathrm{N} 10-\mathrm{C} 29-\mathrm{H} 29$ & 118.5 \\
\hline $\mathrm{F} 4-\mathrm{C} 9-\mathrm{F} 5$ & $106.3(2)$ & $\mathrm{N} 10-\mathrm{C} 30-\mathrm{O} 10$ & $126.7(3)$ \\
\hline
\end{tabular}




\begin{tabular}{|c|c|c|c|}
\hline $\mathrm{F} 4-\mathrm{C} 9-\mathrm{F} 3$ & $107.5(2)$ & $\mathrm{N} 10-\mathrm{C} 30-\mathrm{N} 9$ & $118.7(3)$ \\
\hline $\mathrm{F} 5-\mathrm{C} 9-\mathrm{F} 3$ & $105.6(2)$ & $\mathrm{O} 10-\mathrm{C} 30-\mathrm{N} 9$ & $114.6(2)$ \\
\hline $\mathrm{F} 4-\mathrm{C} 9-\mathrm{C} 7$ & $113.7(2)$ & $\mathrm{O} 9-\mathrm{C} 31-\mathrm{H} 31 \mathrm{~A}$ & 109.5 \\
\hline $\mathrm{F} 5-\mathrm{C} 9-\mathrm{C} 7$ & $111.2(2)$ & $\mathrm{O} 9-\mathrm{C} 31-\mathrm{H} 31 \mathrm{~B}$ & 109.5 \\
\hline $\mathrm{F} 3-\mathrm{C} 9-\mathrm{C} 7$ & $112.0(2)$ & $\mathrm{H} 31 \mathrm{~A}-\mathrm{C} 31-\mathrm{H} 31 \mathrm{~B}$ & 109.5 \\
\hline $\mathrm{N} 3-\mathrm{C} 10-\mathrm{N} 2$ & $118.3(2)$ & $\mathrm{O} 9-\mathrm{C} 31-\mathrm{H} 31 \mathrm{C}$ & 109.5 \\
\hline $\mathrm{N} 3-\mathrm{C} 10-\mathrm{N} 1$ & $123.7(2)$ & $\mathrm{H} 31 \mathrm{~A}-\mathrm{C} 31-\mathrm{H} 31 \mathrm{C}$ & 109.5 \\
\hline $\mathrm{N} 2-\mathrm{C} 10-\mathrm{N} 1$ & $118.0(2)$ & $\mathrm{H} 31 \mathrm{~B}-\mathrm{C} 31-\mathrm{H} 31 \mathrm{C}$ & 109.5 \\
\hline $\mathrm{N} 2-\mathrm{C} 11-\mathrm{N} 4$ & $110.8(2)$ & $\mathrm{O} 10-\mathrm{C} 32-\mathrm{H} 32 \mathrm{~A}$ & 109.5 \\
\hline $\mathrm{N} 2-\mathrm{C} 11-\mathrm{C} 12$ & $130.9(3)$ & $\mathrm{O} 10-\mathrm{C} 32-\mathrm{H} 32 \mathrm{~B}$ & 109.5 \\
\hline $\mathrm{N} 4-\mathrm{C} 11-\mathrm{C} 12$ & $118.2(3)$ & $\mathrm{H} 32 \mathrm{~A}-\mathrm{C} 32-\mathrm{H} 32 \mathrm{~B}$ & 109.5 \\
\hline $\mathrm{C} 13-\mathrm{C} 12-\mathrm{O} 4$ & $128.3(3)$ & $\mathrm{O} 10-\mathrm{C} 32-\mathrm{H} 32 \mathrm{C}$ & 109.5 \\
\hline $\mathrm{C} 13-\mathrm{C} 12-\mathrm{C} 11$ & $117.6(3)$ & $\mathrm{H} 32 \mathrm{~A}-\mathrm{C} 32-\mathrm{H} 32 \mathrm{C}$ & 109.5 \\
\hline $\mathrm{O} 4-\mathrm{C} 12-\mathrm{C} 11$ & $114.1(3)$ & $\mathrm{H} 32 \mathrm{~B}-\mathrm{C} 32-\mathrm{H} 32 \mathrm{C}$ & 109.5 \\
\hline $\mathrm{O} 3-\mathrm{S} 1-\mathrm{N} 1-\mathrm{C} 10$ & $-171.86(18)$ & $\mathrm{C} 16-\mathrm{O} 5-\mathrm{C} 14-\mathrm{N} 4$ & $-178.4(2)$ \\
\hline $\mathrm{O} 2-\mathrm{S} 1-\mathrm{N} 1-\mathrm{C} 10$ & $59.2(2)$ & $\mathrm{C} 11-\mathrm{N} 4-\mathrm{C} 14-\mathrm{N} 5$ & $-0.7(4)$ \\
\hline $\mathrm{C} 8-\mathrm{S} 1-\mathrm{N} 1-\mathrm{C} 10$ & $-56.2(2)$ & $\mathrm{N} 3-\mathrm{N} 4-\mathrm{C} 14-\mathrm{N} 5$ & $178.7(2)$ \\
\hline $\mathrm{C} 10-\mathrm{N} 3-\mathrm{N} 4-\mathrm{C} 11$ & $0.3(2)$ & $\mathrm{C} 11-\mathrm{N} 4-\mathrm{C} 14-\mathrm{O} 5$ & $179.8(2)$ \\
\hline $\mathrm{C} 10-\mathrm{N} 3-\mathrm{N} 4-\mathrm{C} 14$ & $-179.1(2)$ & $\mathrm{N} 3-\mathrm{N} 4-\mathrm{C} 14-\mathrm{O} 5$ & $-0.9(4)$ \\
\hline $\mathrm{O} 7-\mathrm{S} 2-\mathrm{N} 6-\mathrm{C} 26$ & $-176.81(19)$ & $\mathrm{C} 19-\mathrm{O} 6-\mathrm{C} 17-\mathrm{C} 18$ & $175.3(2)$ \\
\hline $\mathrm{O} 8-\mathrm{S} 2-\mathrm{N} 6-\mathrm{C} 26$ & $54.1(2)$ & $\mathrm{O} 6-\mathrm{C} 17-\mathrm{C} 18-\mathrm{F} 6$ & $-61.3(3)$ \\
\hline $\mathrm{C} 24-\mathrm{S} 2-\mathrm{N} 6-\mathrm{C} 26$ & $-60.7(2)$ & $\mathrm{O} 6-\mathrm{C} 17-\mathrm{C} 18-\mathrm{F} 7$ & $55.7(3)$ \\
\hline $\mathrm{C} 26-\mathrm{N} 8-\mathrm{N} 9-\mathrm{C} 27$ & $0.3(2)$ & $\mathrm{C} 17-\mathrm{O} 6-\mathrm{C} 19-\mathrm{C} 20$ & $-4.0(3)$ \\
\hline $\mathrm{C} 26-\mathrm{N} 8-\mathrm{N} 9-\mathrm{C} 30$ & $-178.6(2)$ & $\mathrm{C} 17-\mathrm{O} 6-\mathrm{C} 19-\mathrm{C} 24$ & $177.0(2)$ \\
\hline $\mathrm{C} 3-\mathrm{O} 1-\mathrm{C} 1-\mathrm{C} 2$ & $168.7(2)$ & $\mathrm{O} 6-\mathrm{C} 19-\mathrm{C} 20-\mathrm{C} 21$ & $-176.2(2)$ \\
\hline $\mathrm{O} 1-\mathrm{C} 1-\mathrm{C} 2-\mathrm{F} 1$ & $-56.8(3)$ & $\mathrm{C} 24-\mathrm{C} 19-\mathrm{C} 20-\mathrm{C} 21$ & $2.8(4)$ \\
\hline $\mathrm{O} 1-\mathrm{C} 1-\mathrm{C} 2-\mathrm{F} 2$ & $59.8(3)$ & $\mathrm{C} 19-\mathrm{C} 20-\mathrm{C} 21-\mathrm{C} 22$ & $3.3(4)$ \\
\hline $\mathrm{C} 1-\mathrm{O} 1-\mathrm{C} 3-\mathrm{C} 4$ & $-6.4(3)$ & $\mathrm{C} 20-\mathrm{C} 21-\mathrm{C} 22-\mathrm{C} 23$ & $-4.0(4)$ \\
\hline $\mathrm{C} 1-\mathrm{O} 1-\mathrm{C} 3-\mathrm{C} 8$ & $175.1(2)$ & $\mathrm{C} 21-\mathrm{C} 22-\mathrm{C} 23-\mathrm{C} 24$ & $-1.5(4)$ \\
\hline $\mathrm{O} 1-\mathrm{C} 3-\mathrm{C} 4-\mathrm{C} 5$ & $-174.1(2)$ & $\mathrm{C} 21-\mathrm{C} 22-\mathrm{C} 23-\mathrm{C} 25$ & $173.3(2)$ \\
\hline $\mathrm{C} 8-\mathrm{C} 3-\mathrm{C} 4-\mathrm{C} 5$ & $4.3(3)$ & $\mathrm{O} 6-\mathrm{C} 19-\mathrm{C} 24-\mathrm{C} 23$ & $171.0(2)$ \\
\hline $\mathrm{C} 3-\mathrm{C} 4-\mathrm{C} 5-\mathrm{C} 6$ & $1.0(4)$ & $\mathrm{C} 20-\mathrm{C} 19-\mathrm{C} 24-\mathrm{C} 23$ & $-8.1(3)$ \\
\hline $\mathrm{C} 4-\mathrm{C} 5-\mathrm{C} 6-\mathrm{C} 7$ & $-3.2(4)$ & $\mathrm{O} 6-\mathrm{C} 19-\mathrm{C} 24-\mathrm{S} 2$ & $-11.3(3)$ \\
\hline $\mathrm{C} 5-\mathrm{C} 6-\mathrm{C} 7-\mathrm{C} 8$ & $0.0(3)$ & $\mathrm{C} 20-\mathrm{C} 19-\mathrm{C} 24-\mathrm{S} 2$ & $169.70(19)$ \\
\hline $\mathrm{C} 5-\mathrm{C} 6-\mathrm{C} 7-\mathrm{C} 9$ & $177.8(2)$ & $\mathrm{C} 22-\mathrm{C} 23-\mathrm{C} 24-\mathrm{C} 19$ & $7.4(3)$ \\
\hline $\mathrm{C} 6-\mathrm{C} 7-\mathrm{C} 8-\mathrm{C} 3$ & $5.3(3)$ & $\mathrm{C} 25-\mathrm{C} 23-\mathrm{C} 24-\mathrm{C} 19$ & $-167.0(2)$ \\
\hline $\mathrm{C} 9-\mathrm{C} 7-\mathrm{C} 8-\mathrm{C} 3$ & $-172.4(2)$ & $\mathrm{C} 22-\mathrm{C} 23-\mathrm{C} 24-\mathrm{S} 2$ & $-170.27(18)$ \\
\hline $\mathrm{C} 6-\mathrm{C} 7-\mathrm{C} 8-\mathrm{S} 1$ & $-175.15(17)$ & $\mathrm{C} 25-\mathrm{C} 23-\mathrm{C} 24-\mathrm{S} 2$ & $15.3(3)$ \\
\hline $\mathrm{C} 9-\mathrm{C} 7-\mathrm{C} 8-\mathrm{S} 1$ & $7.2(3)$ & $\mathrm{O} 7-\mathrm{S} 2-\mathrm{C} 24-\mathrm{C} 19$ & $71.2(2)$ \\
\hline $\mathrm{O} 1-\mathrm{C} 3-\mathrm{C} 8-\mathrm{C} 7$ & $171.1(2)$ & $\mathrm{O} 8-\mathrm{S} 2-\mathrm{C} 24-\mathrm{C} 19$ & $-155.77(18)$ \\
\hline $\mathrm{C} 4-\mathrm{C} 3-\mathrm{C} 8-\mathrm{C} 7$ & $-7.5(3)$ & $\mathrm{N} 6-\mathrm{S} 2-\mathrm{C} 24-\mathrm{C} 19$ & $-41.5(2)$ \\
\hline $\mathrm{O} 1-\mathrm{C} 3-\mathrm{C} 8-\mathrm{S} 1$ & $-8.5(3)$ & $\mathrm{O} 7-\mathrm{S} 2-\mathrm{C} 24-\mathrm{C} 23$ & $-111.1(2)$ \\
\hline $\mathrm{C} 4-\mathrm{C} 3-\mathrm{C} 8-\mathrm{S} 1$ & $172.95(18)$ & $\mathrm{O} 8-\mathrm{S} 2-\mathrm{C} 24-\mathrm{C} 23$ & $21.9(2)$ \\
\hline $\mathrm{O} 3-\mathrm{S} 1-\mathrm{C} 8-\mathrm{C} 7$ & $-107.6(2)$ & $\mathrm{N} 6-\mathrm{S} 2-\mathrm{C} 24-\mathrm{C} 23$ & $136.22(19)$ \\
\hline $\mathrm{O} 2-\mathrm{S} 1-\mathrm{C} 8-\mathrm{C} 7$ & $25.5(2)$ & $\mathrm{C} 22-\mathrm{C} 23-\mathrm{C} 25-\mathrm{F} 9$ & $-138.3(2)$ \\
\hline $\mathrm{N} 1-\mathrm{S} 1-\mathrm{C} 8-\mathrm{C} 7$ & $139.78(19)$ & $\mathrm{C} 24-\mathrm{C} 23-\mathrm{C} 25-\mathrm{F} 9$ & $36.3(3)$ \\
\hline $\mathrm{O} 3-\mathrm{S} 1-\mathrm{C} 8-\mathrm{C} 3$ & $72.01(19)$ & $\mathrm{C} 22-\mathrm{C} 23-\mathrm{C} 25-\mathrm{F} 8$ & $97.3(3)$ \\
\hline
\end{tabular}




$\begin{array}{ll}\mathrm{O} 2-\mathrm{S} 1-\mathrm{C} 8-\mathrm{C} 3 & -154.89(17) \\ \mathrm{N} 1-\mathrm{S} 1-\mathrm{C} 8-\mathrm{C} 3 & -40.7(2) \\ \mathrm{C} 6-\mathrm{C} 7-\mathrm{C} 9-\mathrm{F} 4 & -138.2(2) \\ \mathrm{C} 8-\mathrm{C} 7-\mathrm{C} 9-\mathrm{F} 4 & 39.5(3) \\ \mathrm{C} 6-\mathrm{C} 7-\mathrm{C} 9-\mathrm{F} 5 & -18.2(3) \\ \mathrm{C} 8-\mathrm{C} 7-\mathrm{C} 9-\mathrm{F} 5 & 159.5(2) \\ \mathrm{C} 6-\mathrm{C} 7-\mathrm{C} 9-\mathrm{F} 3 & 99.7(3) \\ \mathrm{C} 8-\mathrm{C} 7-\mathrm{C} 9-\mathrm{F} 3 & -82.6(3) \\ \mathrm{N} 4-\mathrm{N} 3-\mathrm{C} 10-\mathrm{N} 2 & 0.0(3) \\ \mathrm{N} 4-\mathrm{N} 3-\mathrm{C} 10-\mathrm{N} 1 & -178.3(2) \\ \mathrm{C} 11-\mathrm{N} 2-\mathrm{C} 10-\mathrm{N} 3 & -0.2(3) \\ \mathrm{C} 11-\mathrm{N} 2-\mathrm{C} 10-\mathrm{N} 1 & 178.2(2) \\ \mathrm{S} 1-\mathrm{N} 1-\mathrm{C} 10-\mathrm{N} 3 & -39.4(3) \\ \mathrm{S} 1-\mathrm{N} 1-\mathrm{C} 10-\mathrm{N} 2 & 142.28(19) \\ \mathrm{C} 10-\mathrm{N} 2-\mathrm{C} 11-\mathrm{N} 4 & 0.4(3) \\ \mathrm{C} 10-\mathrm{N} 2-\mathrm{C} 11-\mathrm{C} 12 & 178.9(3) \\ \mathrm{N} 3-\mathrm{N} 4-\mathrm{C} 11-\mathrm{N} 2 & -0.4(3) \\ \mathrm{C} 14-\mathrm{N} 4-\mathrm{C} 11-\mathrm{N} 2 & 179.0(2) \\ \mathrm{N} 3-\mathrm{N} 4-\mathrm{C} 11-\mathrm{C} 12 & -179.2(2) \\ \mathrm{C} 14-\mathrm{N} 4-\mathrm{C} 11-\mathrm{C} 12 & 0.3(4) \\ \mathrm{C} 15-\mathrm{O} 4-\mathrm{C} 12-\mathrm{C} 13 & 2.7(4) \\ \mathrm{C} 15-\mathrm{O} 4-\mathrm{C} 12-\mathrm{C} 11 & -176.1(2) \\ \mathrm{N} 2-\mathrm{C} 11-\mathrm{C} 12-\mathrm{C} 13 & -178.4(3) \\ \mathrm{N} 4-\mathrm{C} 11-\mathrm{C} 12-\mathrm{C} 13 & 0.1(4) \\ \mathrm{N} 2-\mathrm{C} 11-\mathrm{C} 12-\mathrm{O} 4 & 0.6(4) \\ \mathrm{N} 4-\mathrm{C} 11-\mathrm{C} 12-\mathrm{O} 4 & 179.1(2) \\ \mathrm{O} 4-\mathrm{C} 12-\mathrm{C} 13-\mathrm{N} 5 & -178.9(3) \\ \mathrm{C} 11-\mathrm{C} 12-\mathrm{C} 13-\mathrm{N} 5 & 0.0(4) \\ \mathrm{C} 14-\mathrm{N} 5-\mathrm{C} 13-\mathrm{C} 12 & -0.4(4) \\ \mathrm{C} 13-\mathrm{N} 5-\mathrm{C} 14-\mathrm{O} 5 & -179.8(3) \\ \mathrm{C} 13-\mathrm{N} 5-\mathrm{C} 14-\mathrm{N} 4 & 0.7(4) \\ \mathrm{C} 16-\mathrm{O} 5-\mathrm{C} 14-\mathrm{N} 5 & 2.0(4) \\ & \end{array}$

\begin{tabular}{|c|c|}
\hline $\mathrm{C} 24-\mathrm{C} 23-\mathrm{C} 25-\mathrm{F} 8$ & $-88.1(3)$ \\
\hline $\mathrm{C} 22-\mathrm{C} 23-\mathrm{C} 25-\mathrm{F} 10$ & $-20.8(3)$ \\
\hline $\mathrm{C} 24-\mathrm{C} 23-\mathrm{C} 25-\mathrm{F} 10$ & $153.8(2)$ \\
\hline $\mathrm{N} 9-\mathrm{N} 8-\mathrm{C} 26-\mathrm{N} 7$ & $0.1(3)$ \\
\hline $\mathrm{N} 9-\mathrm{N} 8-\mathrm{C} 26-\mathrm{N} 6$ & $-178.7(2)$ \\
\hline $\mathrm{C} 27-\mathrm{N} 7-\mathrm{C} 26-\mathrm{N} 8$ & $-0.5(3)$ \\
\hline $\mathrm{C} 27-\mathrm{N} 7-\mathrm{C} 26-\mathrm{N} 6$ & $178.4(2)$ \\
\hline $\mathrm{S} 2-\mathrm{N} 6-\mathrm{C} 26-\mathrm{N} 8$ & $-36.0(3)$ \\
\hline $\mathrm{S} 2-\mathrm{N} 6-\mathrm{C} 26-\mathrm{N} 7$ & $145.19(18)$ \\
\hline $\mathrm{C} 26-\mathrm{N} 7-\mathrm{C} 27-\mathrm{N} 9$ & $0.6(2)$ \\
\hline $\mathrm{C} 26-\mathrm{N} 7-\mathrm{C} 27-\mathrm{C} 28$ & $178.6(2)$ \\
\hline $\mathrm{N} 8-\mathrm{N} 9-\mathrm{C} 27-\mathrm{N} 7$ & $-0.7(3)$ \\
\hline $\mathrm{C} 30-\mathrm{N} 9-\mathrm{C} 27-\mathrm{N} 7$ & $178.4(2)$ \\
\hline $\mathrm{N} 8-\mathrm{N} 9-\mathrm{C} 27-\mathrm{C} 28$ & $-178.9(2)$ \\
\hline $\mathrm{C} 30-\mathrm{N} 9-\mathrm{C} 27-\mathrm{C} 28$ & $0.2(3)$ \\
\hline $\mathrm{C} 31-\mathrm{O} 9-\mathrm{C} 28-\mathrm{C} 29$ & $3.1(4)$ \\
\hline $\mathrm{C} 31-\mathrm{O} 9-\mathrm{C} 28-\mathrm{C} 27$ & $-175.4(2)$ \\
\hline $\mathrm{N} 7-\mathrm{C} 27-\mathrm{C} 28-\mathrm{C} 29$ & $-177.5(2)$ \\
\hline $\mathrm{N} 9-\mathrm{C} 27-\mathrm{C} 28-\mathrm{C} 29$ & $0.2(4)$ \\
\hline $\mathrm{N} 7-\mathrm{C} 27-\mathrm{C} 28-\mathrm{O} 9$ & $1.2(4)$ \\
\hline N9-C27-C28-O9 & $178.9(2)$ \\
\hline $\mathrm{O} 9-\mathrm{C} 28-\mathrm{C} 29-\mathrm{N} 10$ & $-179.1(2)$ \\
\hline $\mathrm{C} 27-\mathrm{C} 28-\mathrm{C} 29-\mathrm{N} 10$ & $-0.7(4)$ \\
\hline $\mathrm{C} 30-\mathrm{N} 10-\mathrm{C} 29-\mathrm{C} 28$ & $0.7(4)$ \\
\hline $\mathrm{C} 29-\mathrm{N} 10-\mathrm{C} 30-\mathrm{O} 10$ & $-179.7(2)$ \\
\hline $\mathrm{C} 29-\mathrm{N} 10-\mathrm{C} 30-\mathrm{N} 9$ & $-0.3(4)$ \\
\hline $\mathrm{C} 32-\mathrm{O} 10-\mathrm{C} 30-\mathrm{N} 10$ & $1.5(4)$ \\
\hline $\mathrm{C} 32-\mathrm{O} 10-\mathrm{C} 30-\mathrm{N} 9$ & $-178.0(2)$ \\
\hline $\mathrm{C} 27-\mathrm{N} 9-\mathrm{C} 30-\mathrm{N} 10$ & $-0.2(4)$ \\
\hline $\mathrm{N} 8-\mathrm{N} 9-\mathrm{C} 30-\mathrm{N} 10$ & $178.7(2)$ \\
\hline $\mathrm{C} 27-\mathrm{N} 9-\mathrm{C} 30-\mathrm{O} 10$ & $179.4(2)$ \\
\hline $\mathrm{N} 8-\mathrm{N} 9-\mathrm{C} 30-\mathrm{O} 10$ & $-1.8(4)$ \\
\hline
\end{tabular}

Hydrogen-bond geometry $\left(\AA,{ }^{\circ}\right)$

\begin{tabular}{lllll}
\hline$D-\mathrm{H} \cdots A$ & $D-\mathrm{H}$ & $\mathrm{H} \cdots A$ & $D \cdots A$ & $D-\mathrm{H}^{\cdots} A A$ \\
\hline $\mathrm{C} 1-\mathrm{H} 1 A \cdots \mathrm{F} 10^{\mathrm{i}}$ & 0.99 & 2.48 & $3.293(3)$ & 139 \\
$\mathrm{C} 16-\mathrm{H} 16 C \cdots \mathrm{F} 3^{\mathrm{ii}}$ & 0.98 & 2.40 & $3.185(3)$ & 136 \\
$\mathrm{C} 17-\mathrm{H} 17 B \cdots 8^{\mathrm{i}}$ & 0.99 & 2.40 & $3.102(3)$ & 127 \\
$\mathrm{C} 18-\mathrm{H} 18 \cdots \mathrm{F} 10^{\text {iii }}$ & 1.00 & 2.60 & $3.104(3)$ & 111 \\
$\mathrm{C} 20-\mathrm{H} 20 \cdots \mathrm{F} 9^{i}$ & 0.95 & 2.55 & $3.466(3)$ & 162 \\
$\mathrm{C} 32-\mathrm{H} 32 C \cdots \mathrm{F} 8^{\mathrm{iv}}$ & 0.98 & 2.36 & $3.146(3)$ & 137 \\
\hline
\end{tabular}

Symmetry codes: (i) $x-1, y, z$; (ii) $-x,-y+1,-z+1$; (iii) $x-1, y+1, z$; (iv) $-x+3,-y,-z$. 